\title{
Monte Carlo simulation of a proton therapy beamline for intracranial treatments
}

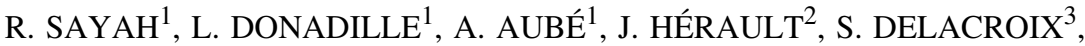 \\ L. DE MARZI ${ }^{3}$, F. STICHELBAUT ${ }^{4}$, I. CLAIRAND ${ }^{1}$
}

(Manuscript received 26 June 2012, accepted 19 December 2012)

\begin{abstract}
A radiation transport code based on a Monte Carlo tool is used to simulate a proton therapy beamline designed to treat paediatric patients with intracranial tumours. The treatments are performed using the IBA gantry at the Proton Therapy Centre of the Institut Curie. The treatment is undertaken at $178.16 \mathrm{MeV}$ using the double scattering technique. The aim of this study is to present the Monte Carlo model of the transported proton beam, beamline and treatment room, as well as the experimental validation of the proton dose distributions calculated by this model. The beamline components and the treatment room are accurately modelled using the Monte Carlo code MCNPX. The proton source at the beamline entrance is defined on the basis of IBA data, measurements and calculations. Measured and calculated relative proton dose distributions in a water phantom are compared for the validation. Depth dose profiles, including pristine Bragg peaks and a spread-out Bragg peak, and lateral dose profiles are studied. A general good agreement was found between calculated and measured distributions with discrepancies of less than $\mathbf{2} \mathbf{~ m m}$. Relative proton dose distributions are therefore considered to be correctly described by our simulated geometry and proton source parameters. The Monte Carlo simulation will be used subsequently for radiation protection purposes: calculation of secondary neutron doses received by treated patients of different ages.
\end{abstract}

Keywords: proton therapy / dosimetry / Monte Carlo

\section{Introduction}

Proton therapy is a radiation therapy technique currently practised in about 30 centres worldwide (PTCOG, 2010). Protons are heavy charged particles with interesting ballistic properties, i.e. small lateral dispersion in biological tissue compared with photons, and limited range and shape of the depth dose distribution characterised by a maximum dose deposition at a defined depth: the Bragg peak. Therefore, proton therapy can accurately treat tumours by delivering high dose levels to the target volume while saving the healthy surrounding tissues (Bonnet, 1993; Sisterson, 1995; Olsen et al., 2007).

\footnotetext{
1 IRSN, Laboratoire de Dosimétrie des Rayonnements Ionisants, PRP-HOM/SDE/LDRI - BP17, 92262 Fontenay-aux-Roses Cedex, France.

2 Centre Antoine Lacassagne (CAL) - Cyclotron biomédical, 227 avenue de la Lanterne, 06200 Nice, France.

3 Institut Curie - Centre de Protonthérapie d'Orsay (ICPO) - Campus universitaire bâtiment 101, 91898 Orsay, France.

4 IBA, 1348 Louvain-la-Neuve, Belgium.
} 
The Proton Therapy Centre of the Institut Curie (ICPO) in France has treated over 5000 patients since its creation in 1991. In 2010, the old synchrocyclotron was replaced by a new cyclotron, and a new gantry room was installed by the Ion Beam Applications (IBA) Company, in addition to the other existing treatment rooms in order to respond to the increasing number of patients and the diversification of therapeutic indications. Passive beam scattering is the delivery technique currently used for all treatments at the ICPO. The active beam scanning technique will be adopted later in the gantry room.

In the framework of collaboration between the French Institute for Radiation Protection and Nuclear Safety (IRSN) and the ICPO, a research programme is devoted to the evaluation of secondary neutron doses received by healthy tissues in patients. An initial study focused on ophthalmological treatments undertaken at $75 \mathrm{MeV}$ (Martinetti et al., 2009). The study was extended to intracranial treatments, which are performed in the new gantry room at energies close to $200 \mathrm{MeV}$. The same procedure was adopted for the two kinds of treatments: a Monte Carlo model of the treatment room and the beamline components are developed and their validity is checked against experimental measurements. The validated model is then used to calculate secondary neutron doses in anthropomorphic phantoms introduced into the Monte Carlo model. Only the passive scattering technique is involved in both studies.

The Monte Carlo technique is widely used in radiation therapy fields. In proton therapy, it has been validated by several published studies. With an accurate Monte Carlo simulation of the beamline, authors have reported a good agreement between calculated and measured proton dose distributions (Paganetti et al., 2004; Hérault et al., 2005; Newhauser et al., 2005; Polf et al., 2007; Martinetti et al., 2009; Stankovskiy et al., 2009). Recent literature even suggests using Monte Carlo methods in clinical proton therapy applications (Hérault et al., 2007; Paganetti et al., 2008). In fact, clinical treatment planning systems are based on a parameterisation of measured proton distributions and therefore they suffer from approximations in modelling particle transport physics. On the contrary, Monte Carlo tools follow beam propagation in space by considering all particle interactions and energy losses on the basis of physical models and/or reaction cross-section libraries. Monte Carlo methods also turn out to be powerful in complex situations where measurements are difficult to achieve. They also have many other applications in proton therapy: verification of shielding design efficiency (Newhauser et al., 2002), assessment of secondary doses received by proton therapy patients (Agosteo et al., 1998; Jiang et al., 2005; Zacharatou Jarlskog and Paganetti 2008) and neutron fluence and ambient dose equivalent calculations (Zheng et al., 2008; Perez-Andujar et al., 2009). It should be noted 
that each Monte Carlo model must first be carefully validated with experimental data in order to verify the accuracy of results.

The aim of this study is to present the Monte Carlo simulation model of the new IBA gantry room at the ICPO and the experimental validation of the proton dose distributions in a water phantom. Firstly, a comparison is performed between experimental and calculated relative depth dose distributions for modulated and non-modulated beams and, secondly, measured and calculated relative lateral dose profiles are also compared in modulated conditions at two different depths in the water phantom.

\section{Material and methods}

\subsection{Geometry simulation}

\subsubsection{Beamline components}

The IBA cyclotron produces protons with a fixed energy of $230 \mathrm{MeV}$ and a maximum intensity of $500 \mathrm{nA}$. After it exits the cyclotron, the proton beam passes through the Energy Selection System (ESS), which consists of different degraders and magnets in order to achieve and select the desired energy before the beam reaches one of the different treatment rooms. Since the beamline configuration in the gantry varies between different kinds of treatments, depending on the tumour's volume and localisation, in the present study we focused on a paediatric treatment using the double scattering technique: a craniopharyngioma treatment undertaken at $178.16 \mathrm{MeV}$. Consequently, our model corresponds to the configuration required for this kind of treatment only.

The beamline components consist of different absorbers, beam shaping and monitoring devices that achieve an accurate and homogeneous dose distribution over the target volume at the desired depth. All beamline or nozzle elements were accurately modelled on the basis of original drawings from IBA. A schematic presentation of the beamline components is shown in Figure 1, where distances between the different elements are indicated. The nozzle length is about $285 \mathrm{~cm}$. The scanning magnets ( $\mathrm{X}$ and $\mathrm{Y}$ ) are only used in the beam scanning technique and therefore they were not modelled in our study. A similar IBA nozzle design was described by Paganetti et al. (2004). All the nozzle components as modelled by the Monte Carlo tool are shown in Figure 2.

The first modelled component is the first ionisation chamber (IC1); it measures the beam profile and position at the nozzle entrance. The beam then passes through the first scatterer. It consists of several foils that can be combined in different 


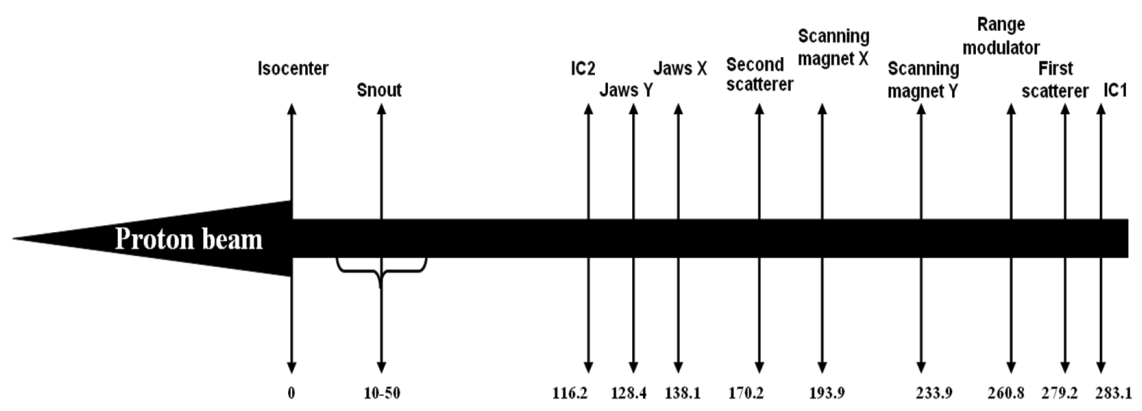

Figure 1 - Schematic view of the IBA beamline components. The lower numbers indicate the distances in cm between each component and the isocenter. The snout is the only movable part that can be placed between 10 and $50 \mathrm{~cm}$ from the isocenter. IC1 and IC2 refer to the first and the second ionization chambers, respectively.
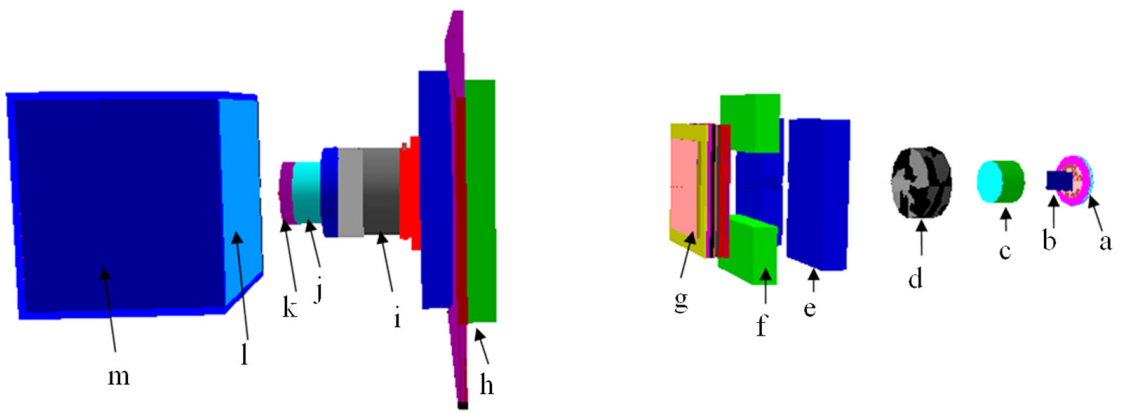

Figure 2-(Color online) 3D view of the nozzle model. The nozzle frame was removed for better visualization of the different components: (a) first ionization chamber, (b) first scatterer, (c) range modulator, $(d)$ second scatterer, $(e) X j a w s,(f) Y j a w s,(g)$ second ionization chamber, (h) snout translation system, (i) snout, $(j)$ personal collimator, $(k)$ personal compensator, (l) water, (m) water phantom.

ways, depending on the desired range in the patient, so every foil can be placed in or out of the beam trajectory during irradiation. In our chosen treatment configuration, six tantalum foils are placed in the beam to achieve a range of $10.5 \mathrm{~cm}$ in the patient. They were modelled as $2.8 \mathrm{~cm} \times 2.3 \mathrm{~cm}$ rectangular foils with a total thickness of $2.59 \mathrm{~mm}$. 


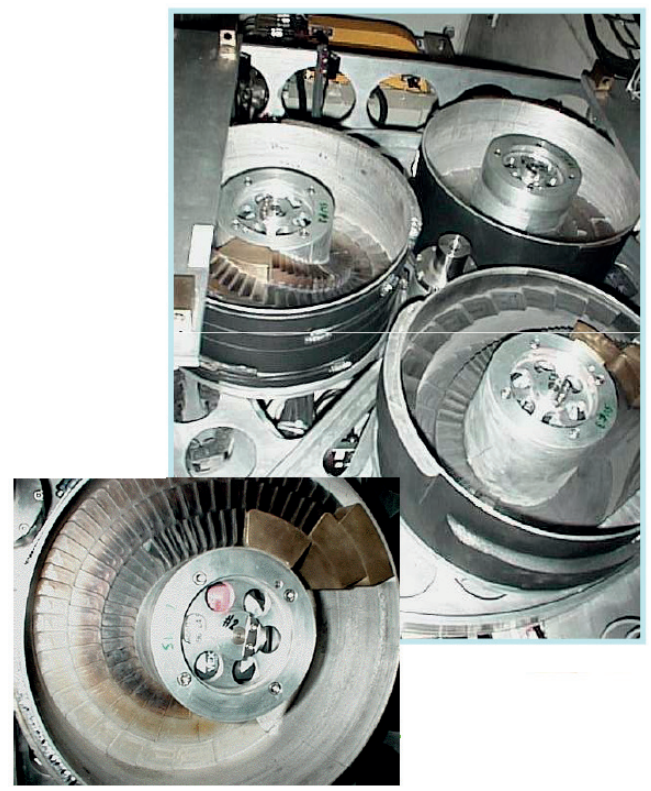

Figure 3-(Color online) The IBA range modulators assembly. The upper figure shows the whole assembly made of three wheels. The lower figure presents one of these wheels that hold three tracks; each track presents a range modulator made of many steps with different thicknesses.

Downstream of the first scatterer, the beam reaches the range modulator. It consists of three rotating wheels with three tracks on each wheel. Every track is made of several steps of various thicknesses, and each step has two layers of superimposed lead and lexan. The IBA range modulator assembly is presented in Figure 3. Only one track is placed in the beam path for a given treatment configuration. As the beam passes through the rotating track, protons undergo variable amounts of range shifting depending on the crossed step thickness. Pristine Bragg peaks are thus created at different depths: their sum yields a SpreadOut Bragg Peak (SOBP). In addition, the beam current is continuously modulated as a function of the rotating angle in order to achieve an acceptable SOBP flatness; this is the so-called beam current modulation (BCM). In the simulation, the range modulator was modelled by two superimposed 5-cm-radius cylinders with a finite thickness of lead for the first and lexan for the second cylinder. The method adopted to simulate the modulation is detailed later on.

The proton beam reaches the second scatterer with a normal transverse shape. In order to achieve a broad uniform beam, the protons near the beam axis should 


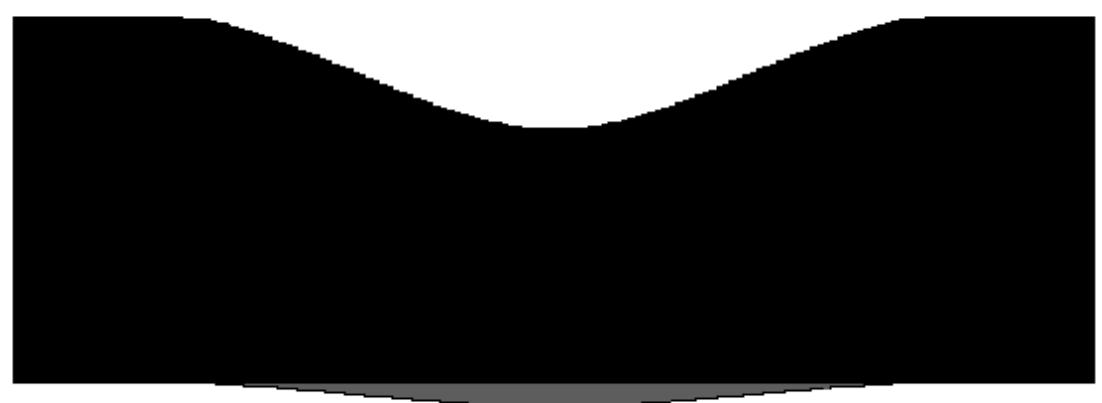

Figure 4 - The beamline second scatterer as modelled in MCNPX. The upper part in black presents the lexan component and the lower grey part presents the lead component.

experience a stronger scattering than those further away from the beam axis. This explains the second scatterer's design, an association of lead and lexan: the lead, a highly scattering material, has a thickness decreasing from the disk's centre to the edges; however, in order to ensure an energy loss independent of the disk radius, the lexan is used as a low scattering material with an increasing thickness from the disk's centre to the edges. Both the lead and lexan components of the second scatterer used in the chosen treatment were modelled in MCNPX with 111 and 115 embedded cylinders, respectively. The height of each cylinder was defined according to the material's thickness at the corresponding radius. Figure 4 shows the second scatterer as modelled in MCNPX.

In our treatment configuration, the variable collimators have an opening of $15.6 \mathrm{~cm}$ in the $X$ direction and $15.7 \mathrm{~cm}$ in the $Y$ direction. The $\mathrm{X}$ jaws are made of nickel while the $\mathrm{Y}$ jaws are made of brass.

The second ionisation chamber (IC2) monitors beam flatness and uniformity. It also measures the dose before the beam reaches the patient. The chamber is made of different foils that were accurately modelled.

The snout is a movable part of the nozzle that holds the patient's specific collimator and range compensator. A translation system helps position the snout at the desired distance from the patient; an air gap of $50 \mathrm{~mm}$ was chosen in our configuration. The snout can have three different dimensions but the 100-mmradius size was chosen in our study since it is the most frequently used in paediatric treatments. The translation system made of aluminium and the snout made of brass 
were both modelled. A 55-mm-diameter collimator and a 3-mm-thick flat compensator were adopted for this study. The collimator is made of brass and the compensator of Plexiglas.

The nozzle components from the first ionisation chamber to the snout's base were enclosed in an iron nozzle frame of $1.5 \mathrm{~cm}$ thickness.

The water phantom where the proton dose distributions were measured was included in the Monte Carlo model, with exterior dimensions of $65 \mathrm{~cm} \times 65 \mathrm{~cm} \times$ $65 \mathrm{~cm}$ and filled with water. The phantom's walls are made of Plexiglas with a $2.5 \mathrm{~cm}$ thickness, except for the top side. The isocentre is located at 8-cm-depth in the phantom. The IBA gantry can rotate $360^{\circ}$ around the patient. During all the proton dose validations presented in this paper, the gantry was vertically placed at an angle of $0^{\circ}$, i.e. above the water phantom.

Finally, the beam stop made of polyethylene and the counter-weight made of steel were also modelled downstream of the nozzle since they could be a source of secondary neutrons.

\subsubsection{The treatment room simulation}

The treatment room was modelled on the basis of engineering drawings. The complicated metallic rotating structure of the gantry was not included in the model; since the proton beam does not directly interact with this structure, it should not have any impact on the proton doses. We also considered that the rotating structure has little influence on the secondary neutron doses received by the patients. This assumption will be confirmed in a later step where an experimental validation of the secondary neutron doses calculated by our model will be performed. However, the walls, ceiling, floor and the entrance maze, all essentially made of concrete, were taken into account. A visualisation of the modelled treatment room in both the horizontal and the vertical planes containing the isocentre is presented in Figure 5. Figure 5a corresponds to the horizontal plane of the treatment room with the gantry horizontally oriented at $270^{\circ}$ for a better visualisation of the beamline, although this orientation was not used during the proton dose validation presented in this paper. The concrete's composition was provided by IBA. It should be noticed that this part of the geometry does not influence the proton dose distributions presented here. Nevertheless, it was included in our simulation since our final purpose is to calculate doses from secondary neutrons that can also be generated in the room materials. 


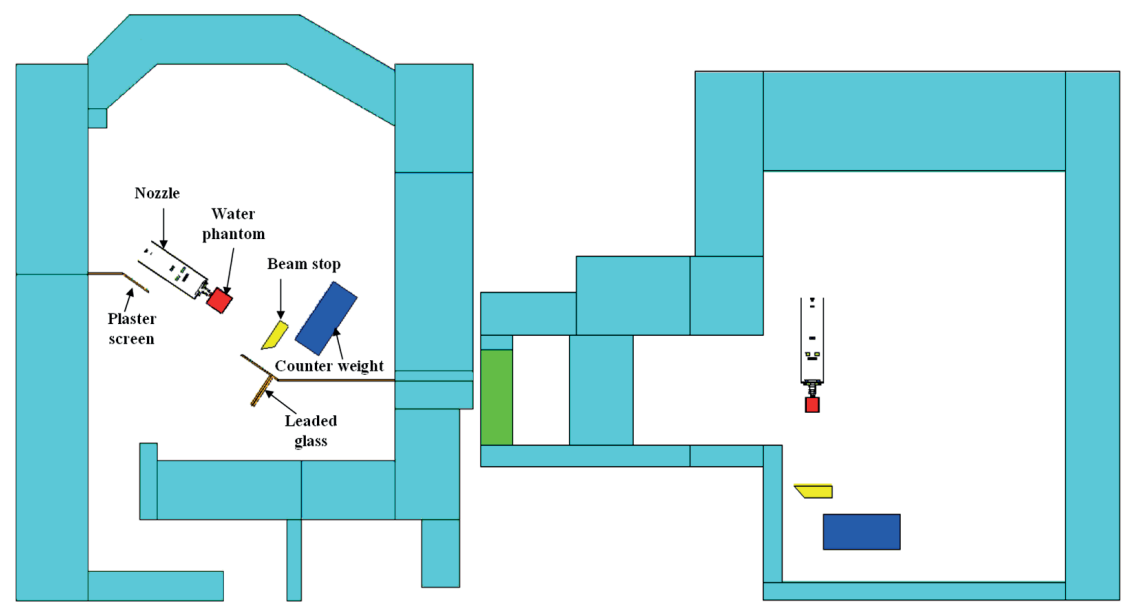

(a)

(b)

Figure 5 - (Color online) $2 D$ views of the full geometry; in the horizontal plane (a) with the gantry placed at $270^{\circ}$ and in the vertical plane, (b) with the gantry rotated at $0^{\circ}$ (orientation used for the proton dose validations); both views contain the isocenter. The counter weight and the beam stop also rotate with the gantry.

\subsection{The proton beam source}

As already mentioned, a paediatric craniopharyngioma treatment was adopted in this study. The mean proton energy at the nozzle entrance is $E=178.16 \mathrm{MeV}$ with a range in water of $21.27 \mathrm{~cm}$. The proton beam source was modelled at the level of the first ionisation chamber, i.e. the nozzle's entrance. Its characteristics must be accurately described in the Monte Carlo model in order to obtain a good agreement between calculations and measurements. On the basis of the IBA data, the beam energy at the nozzle entrance is normally distributed around $E$ with $\sigma_{E} / E=0.6 \%$, with $\sigma_{E}$ as the standard deviation. No information was given on the distribution's limits, so they were approximated with an ad hoc method: the higher and lower beam energy limits were modified in each simulation until good agreement was obtained between calculated and measured distributions. These adjustments were only performed on the deepest Bragg peak corresponding to the first modulator step without any personal compensator. The beam lateral shape at the nozzle entrance was determined using the first ionisation chamber measurements. In order to define the angular distribution, the nozzle components and the water phantom were removed from the beam path, and the beam spot size was measured by radiochromic films placed perpendicularly to the beam axis at three different positions: $260 \mathrm{~cm}$ and $53.65 \mathrm{~cm}$ upstream, and $46.65 \mathrm{~cm}$ 
MONTE CARLO SIMULATION OF A PROTON THERAPY BEAMLINE

downstream of the isocentre. The variation of the beam spot size between these measurements helped determine the beam angular divergence.

\subsection{Measurements}

Proton dose distributions were measured in the water phantom, which is equipped with an automatic scanning system. Two kinds of ionisation chambers were used. The depth dose distributions were measured by a PPC05 plane parallel chamber that has an active volume of $0.05 \mathrm{~cm}^{3}$ and the transverse profiles were measured by a CC01 compact chamber with a cavity volume of $0.01 \mathrm{~cm}^{3}$ (IBA, 2007). Both chambers have been extensively tested to meet the highest criteria in radiation therapy dosimetry. They provide measurements with a maximum uncertainty of $3 \%$.

Non-modulated and modulated depth dose distributions were measured. First, the range modulator wheel was kept static and the pristine Bragg peaks corresponding to each of the modulator steps involved in the treatment were measured separately. Then, the clinical SOBP was measured in our defined treatment configuration.

The transverse dose profiles were only measured in modulated conditions. The measurements were performed in the two transverse directions at 0.5 and $7 \mathrm{~cm}$ depth in the water phantom.

\subsection{Monte Carlo calculations}

\subsubsection{MCNPX code}

The simulations were performed using the Monte Carlo code MCNPX (version 26c) (Hendricks et al., 2006). Different types of particles can be transported, including protons and neutrons. Proton absorbed doses calculated with MCNPX have been validated with measurements in different studies (Herault et al., 2005; Newhauser et al., 2005; Polf et al., 2007; Martinetti et al., 2009).

\subsubsection{Particle transport}

Protons, neutrons and photons were transported in all the simulations. It is considered that all other particles deposit their energy locally. To define the probabilities of particle interactions, a choice can be made between physical models and evaluated cross-section libraries. For protons and neutrons, we used the LA150H and the LA150N libraries (Chadwick et al., 1999), respectively, from which data are available up to a maximum energy of $150 \mathrm{MeV}$. We used the "Mix and Match" option to use physical models, notably the Bertini model (Bertini, 1968) above this energy 
and for all other energies where the cross-section tables are not available. The water mean ionisation energy is defined in MCNPX with a value of $75.3 \mathrm{eV}$. The proton energy straggling was described by the Vavilov theory (Vavilov, 1957). When this model was unspecified, calculations were performed using the Continuous Slowing Down Approximation (CSDA). Multiple Coulomb Scattering (MCS) is implemented in MCNPX and based on Rossi's theory (Rossi and Greisen, 1941), considering a normal distribution for angular deflection. MCS is not optional in MCNPX; there are no parameters to turn it off. The MCPLIB04 (White, 2002) cross-section library was used for photons.

\subsubsection{Scoring method}

The relative depth dose distributions were calculated using cylindrical mesh tallies. The length of the mesh grid elements along the beam axis was not constant: it decreased from $0.06 \mathrm{~cm}$ to $0.02 \mathrm{~cm}$ in the regions where a strong dose variation was expected. $6 \times 10^{6}$ source protons were tracked in each simulation in order to achieve a statistical uncertainty of less than $2 \%$.

Rectangular mesh tallies were used to calculate relative lateral dose profiles at two depths in the water phantom: 0.5 and $7 \mathrm{~cm}$. The calculation step of $0.4 \mathrm{~cm}$ in the plateau region was decreased to $0.1 \mathrm{~cm}$ near the penumbra to get accurate distributions. In order to achieve an uncertainty of less than $2 \%$ on the plateau, $10 \times 10^{6}$ source protons were tracked.

Simulations were parallelised on three dual $3.0 \mathrm{GHz}$ processor nodes. The computation time required for the simulation of $10^{6}$ histories was approximately one hour.

\subsection{Simulation of the modulation effect}

As already mentioned, the proton beam is experimentally modulated in depth by a rotating range modulator that contains several steps of varying thicknesses. MCNPX does not simulate variable geometries in time; therefore, an alternative method should be used to calculate modulated distributions. As previously adopted by some authors (Hérault et al., 2005; Newhauser et al., 2005), we performed several simulations, each one corresponding to each of the crossed steps in order to reproduce the modulation. In every simulation the modulator was modelled by two superimposed cylinders with each having the exact thickness of lead and lexan as the simulated step. A weighting factor to apply to each of the simulation results is calculated on the basis of the steps' angular opening and the BCM. The weighted results are then added to obtain the modulated profile. 
The applied formula is: $D(z)=\sum_{i} p i \times D i(z)$ where $D(z)$ is the total dose at the depth $z, p i$ is the weighting factor calculated for the ith step, and $\operatorname{Di}(z)$ is the absorbed dose at the depth $z$ when only considering the pristine Bragg peak resulting from the $i$ th step. This method was applied to calculate both the SOBP and modulated lateral dose profiles. The MCNPX output files corresponding to each of the modulator steps provide the calculated dose in every voxel of the mesh tally grid, and the associated relative uncertainty, $r_{i}=\frac{\Delta D_{i}}{D_{i}}$. Subsequently, the uncertainty on the dose $D(z)$ resulting from the different modulator steps is given by:

$$
\Delta D^{2}=\sum_{i}\left(\frac{\partial D(z)}{\partial D_{i}(z)}\right)^{2} \Delta D_{i}^{2}=\sum_{i} p_{i}^{2}\left(D_{i} r_{i}\right)^{2} \Leftrightarrow \Delta D=\sqrt{\sum_{i} p_{i}^{2}\left(D_{i} r_{i}\right)^{2}}
$$

the sum being performed on the different modulator steps $i$ involved in the treatment configuration.

The full angular range covered by the beam during any single rotation of the modulator was first measured using a radiographic film attached to the modulator. The steps crossed by the beam in our treatment configuration could therefore be visualised.

It should be mentioned that the simulation of the spread-out Bragg peak using a weighting of the individual pristine Bragg peaks should give similar results to the simulation of the rotation of the range modulator because the weighting factors incorporate the angular opening of each modulator step, i.e. the duration required by the proton beam to pass through each step. In addition, the range modulator is located at the nozzle entrance where the proton beam is narrow; hence, ignoring the beam covering of two steps at the same time is an acceptable approximation.

\subsection{Comparison method}

\subsubsection{Relative depth dose distributions}

Relative depth dose profiles calculated with MCNPX were compared with measurements in both cases of pristine Bragg peaks and the SOBP.

Every non-modulated distribution was normalised at the depth of the maximum dose, as usually done in previous studies (Paganetti et al., 2004; 
Newhauser et al., 2005; Koch et al., 2008; Martinetti et al., 2009). The simulations were compared with the measurements using several characteristics of the beam profiles:

- the range, which was chosen as the distal (d) depth of $90 \%$ of the maximum dose $\left(Z_{d 90}\right)$,

- two field widths chosen as the distance between the proximal $(p)$ and distal depths of $90 \%$ and $50 \%$ of the maximum dose $\left(Z_{p 90}-Z_{d 90}\right.$ and $\left.Z_{p 50}-Z_{d 50}\right)$, and

- the penumbra width, the distance between the distal depths of $80 \%$ and $20 \%$ of the maximum dose $\left(Z_{d 80}-Z_{d 20}\right)$.

The SOBP was normalised to the mean dose calculated on the plateau region. The ratio between the calculated SOBP and the measured one for each measurement depth was analysed for better comparison. In addition, the differences between the calculated and measured:

- range $\left(Z_{d 90}\right)$,

- modulation width chosen here as $\left(Z_{p 95}-Z_{d 95}\right)$, and

- penumbra width $\left(Z_{d 80}-Z_{d 20}\right)$ were established.

The different parameters considered for the comparison of the depth dose distributions are shown in Figure 6.

\subsubsection{Relative transverse distributions}

Calculated relative lateral dose profiles were also compared with measurements at two depths in the water phantom and for both transverse directions. All of the lateral profiles were normalised to the mean dose in the plateau region. The comparison was made by calculating the ratio of the calculated to the measured profiles. Furthermore, the profile and the penumbra widths were also compared. Two field widths were compared: the distances between the two $90 \%$ and $50 \%$ dose levels, respectively. The penumbra width was considered as the distance between the $80 \%$ and $20 \%$ dose levels. The parameters considered for the comparison of the lateral profiles are visualised in Figure 7.

\section{Results and discussion}

\subsection{Proton beam source}

Proton beam energy is normally distributed with a mean energy and a full width at half maximum FWHM equal to $178.16 \mathrm{MeV}$ and $2.52 \mathrm{MeV}$, respectively. The sampling energy limits were adjusted so that good agreement was obtained between the calculated and measured profiles for the first modulator step only, 
MONTE CARLO SIMULATION OF A PROTON THERAPY BEAMLINE

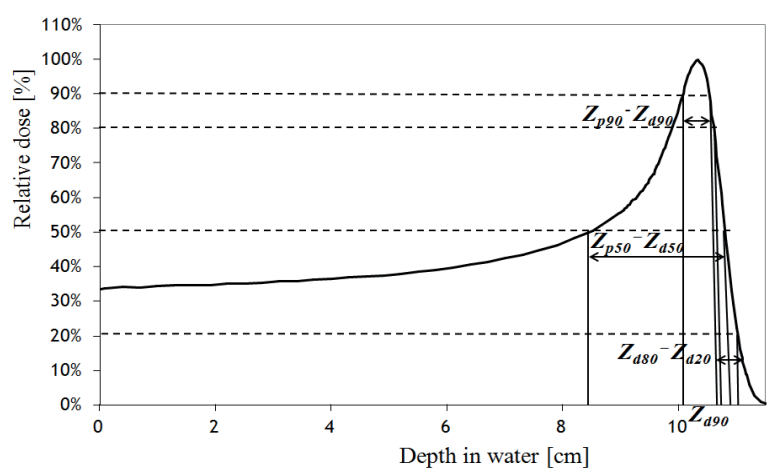

(a)

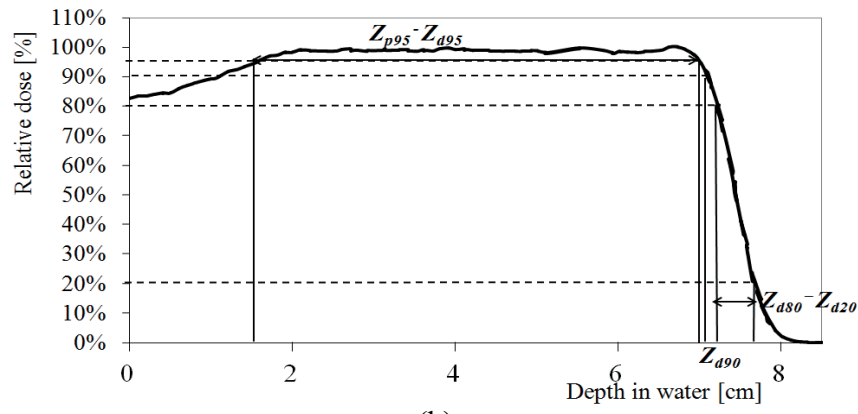

(b)

Figure 6 - The parameters considered for the comparison of the calculated to the measured proton depth dose distributions. (a) The parameters relative to the pristine Bragg peaks, (b) the parameters relative to the spread-out Bragg peak.

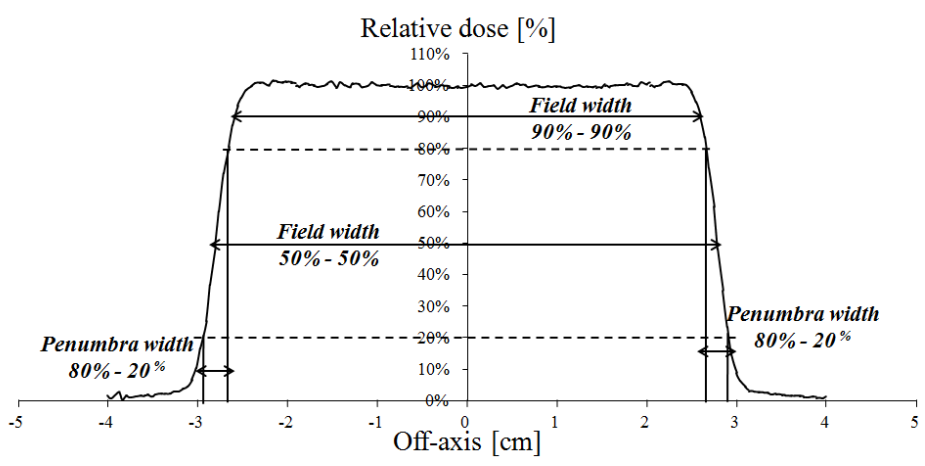

Figure 7 - The parameters considered for the comparison of the calculated to the measured proton lateral dose profiles. 


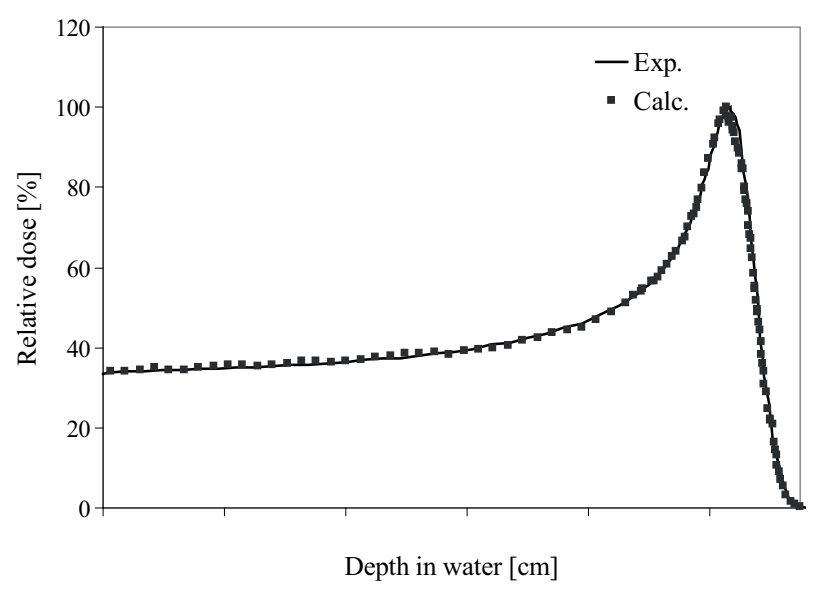

Figure 8 - Deepest Bragg peak corresponding to the first modulator step. Comparison between measured (Exp.) and calculated (Calc.) depth dose profiles; calculations are performed with the adjusted beam energy, position and angular distributions.

i.e. the deepest Bragg peak. The energy limits have an important influence on the Bragg peak shape, especially on the penumbra width and the relative entrance dose. A satisfactory result was obtained when the energy was sampled between 176.10 and $179.43 \mathrm{MeV}$. The deepest Bragg peak calculated with this energy distribution is compared with the measured peak in Figure 8. The beam spot at the entrance is normally shaped in both transverse directions with a FWHM of $1.21 \mathrm{~cm}$ and $0.98 \mathrm{~cm}$ along the $X$ and $Y$ axes, respectively. Relying on the film measurements performed at three different positions, the beam angular deflection was approximated by an isotropic distribution restricted to a cone of $6 \mathrm{mrad}$ (half opening angle) around the beam axis.

\subsection{Modulation}

The film attached to the range modulator during irradiation clearly showed that only 10 steps out of 22 are involved during irradiation in order to achieve a modulation width of $56 \mathrm{~mm}$ (defined as $Z_{p 95}-Z_{d 95}$ ). This result, together with the known BCM values, allowed us to determine the weighting factors to apply to the pristine Bragg peaks resulting from each simulation. Table I shows the lead and lexan thicknesses of each of the modulator steps involved in our treatment configuration as well as the corresponding weighting factors. 
TABLE I

Lexan and lead thicknesses of each of the modulator steps and the calculated steps' weighting factors; the weighting factors' sum is equal to unity.

\begin{tabular}{cccc}
\hline Modulator step & \multicolumn{2}{c}{ Modulator thickness $(\mathbf{c m})$} & Weighting factor \\
\hline 1 & Lexan thickness & Lead thickness & \\
\hline 2 & 0.106 & 0.317 & 0.382 \\
3 & 0.791 & 0.297 & 0.145 \\
4 & 0.152 & 0.269 & 0.099 \\
5 & 2.164 & 0.256 & 0.079 \\
6 & 2.887 & 0.229 & 0.076 \\
7 & 3.531 & 0.216 & 0.065 \\
8 & 4.209 & 0.197 & 0.056 \\
9 & 4.918 & 0.172 & 0.048 \\
10 & 5.562 & 0.160 & 0.045 \\
\hline
\end{tabular}

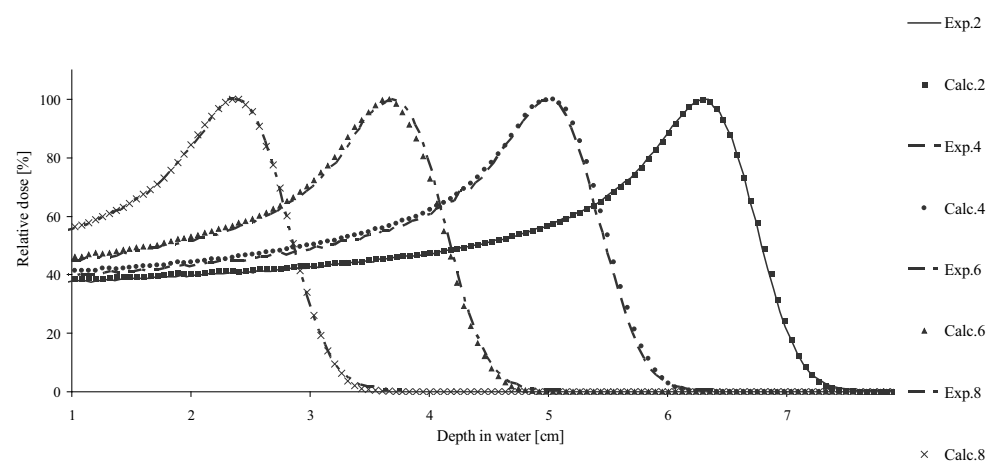

Figure 9 - Measured (Exp.) and calculated (Calc.) relative depth dose profiles. The profiles corresponding to four modulator steps: 2, 4, 6 and 8 are only shown for a better visualisation.

\subsection{Relative depth dose profiles}

\subsubsection{Pristine Bragg peaks}

Ten Bragg peaks corresponding to the ten modulator steps involved in our treatment configuration were compared. For better visualisation, only the measured and calculated peaks corresponding to the second, forth, sixth and eighth modulator steps are shown in Figure 9. Quantitative comparisons in terms of range, peak and penumbra widths for the ten peaks are made in Table II. The 


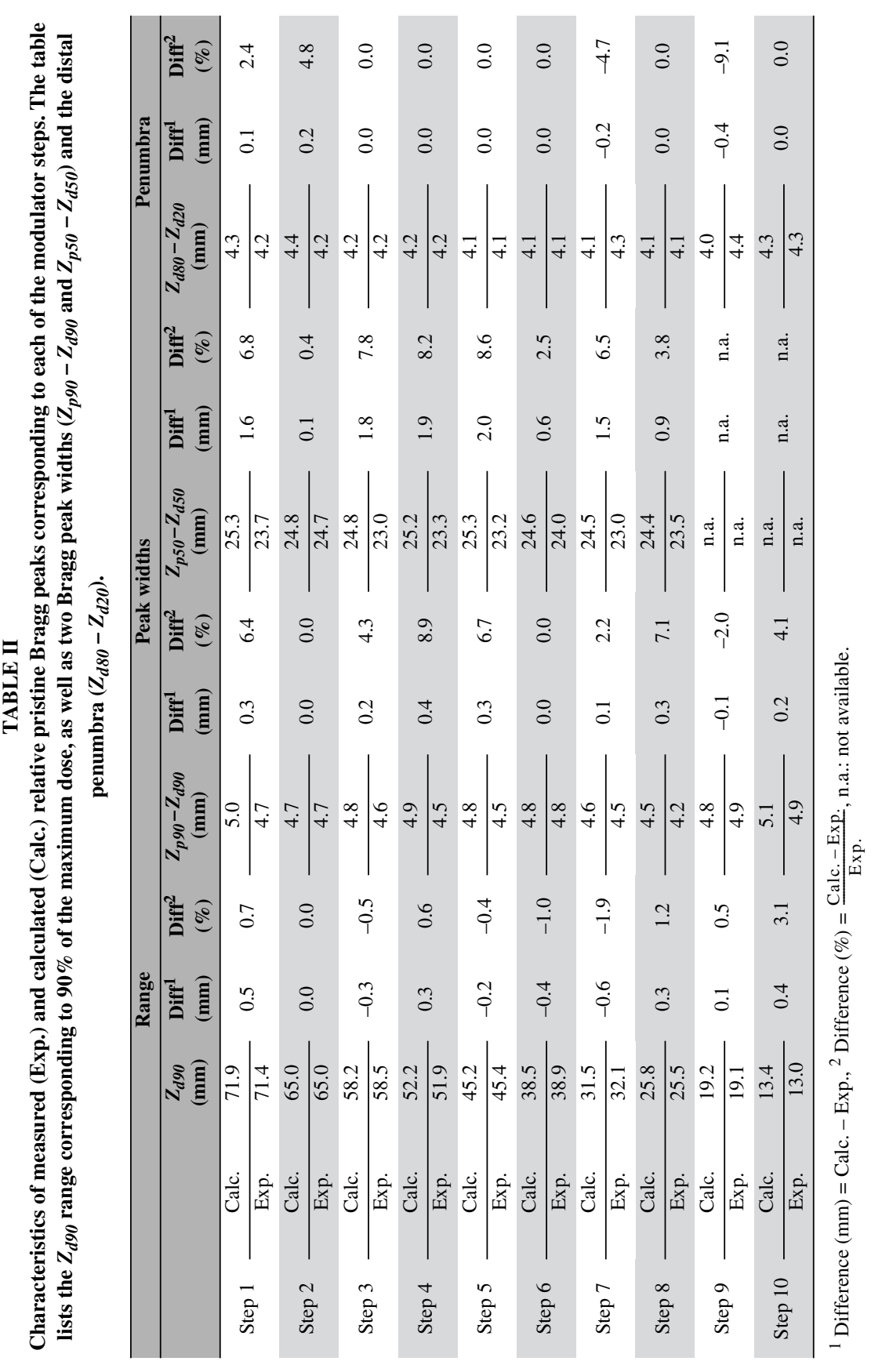


maximum range $\left(Z_{d 90}\right)$ difference is $0.6 \mathrm{~mm}$. The comparison of the peak widths reveals relatively larger differences among calculations and measurements for $Z_{p 90}-Z_{d 90}$, reaching $8.9 \%$ in sector 4 of the modulator, though this difference corresponds to only $0.4 \mathrm{~mm}$. The field width $Z_{p 50}-Z_{d 50}$ was not calculated for the ninth and tenth sectors since the entrance dose levels for these two peaks are higher than the $50 \%$ maximum dose. For the other sectors, the difference in the field width $Z_{p 50}-Z_{d 50}$ is less than $9 \%$, which corresponds to $2 \mathrm{~mm}$. A similar agreement is found for the penumbra: although the relative difference can reach $9.1 \%$, this corresponds to an absolute difference limited to $0.4 \mathrm{~mm}$.

Those discrepancies found between the calculated and the measured depth dose distributions are mainly explained by the uncertainties on the components' thicknesses used to simulate the beamline geometry. In addition, the beam source parameters, especially the upper and lower energy limits, were determined by adjusting the simulated native Bragg peak corresponding to the first modulator step (the thinnest step), to the measured one. The nine peaks corresponding to the other steps were not considered for the adjustment. Subsequently, the beam source parameters are also affected by uncertainties, which may explain the differences found. However, the results show a general good agreement between experimental and calculated Bragg peaks.

\subsubsection{Spread-out Bragg peak}

Calculated and measured SOBPs are plotted in Figure 10. The corresponding ratio between the calculated and the measured SOBP is superimposed on the same

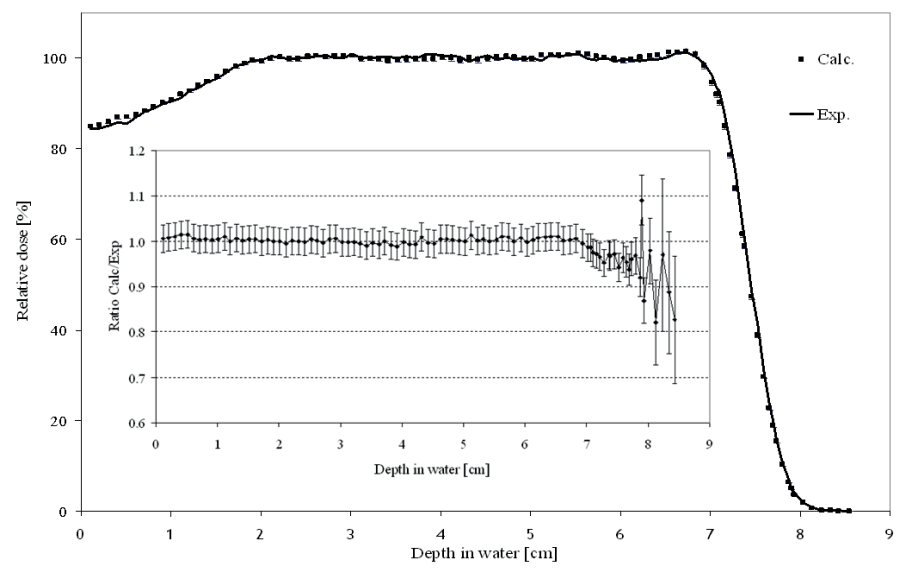

Figure 10 - Measured (Exp.) and calculated (Calc.) relative Spread-out Bragg Peak in water. The ratio between calculated and measured relative SOBP with the uncertainties is superimposed on the SOBP graph for better visualization. 
TABLE III

Characteristics of measured (Exp.) and calculated (Calc.) Spread-out Bragg peaks. The table lists the range $\left(Z_{d 90}\right)$, the modulation width $\left(Z_{p 90}-Z_{d 90}\right)$ and the distal penumbra $\left(Z_{d 80}-Z_{d 20}\right)$.

\begin{tabular}{|c|c|c|c|c|}
\hline & & & Diff $^{1}(\mathrm{~mm})$ & $\operatorname{Diff}^{2}(\%)$ \\
\hline \multirow{2}{*}{ Range $Z_{d 90}(\mathrm{~mm})$} & Calc. & 71.0 & \multirow[t]{2}{*}{-0.3} & \multirow[t]{2}{*}{-0.4} \\
\hline & Exp. & 71.3 & & \\
\hline \multirow{2}{*}{$\begin{array}{l}\text { Modulation width }\left(Z_{p 95}-Z_{d 95}\right) \\
(\mathrm{mm})\end{array}$} & Calc. & 56.0 & \multirow[t]{2}{*}{0.1} & \multirow[t]{2}{*}{0.2} \\
\hline & Exp. & 55.9 & & \\
\hline \multirow{2}{*}{ Penumbra $\left(Z_{d 80}-Z_{d 20}\right)(\mathrm{mm})$} & Calc. & 4.6 & \multirow[t]{2}{*}{0.0} & \multirow[t]{2}{*}{0.0} \\
\hline & Exp. & 4.6 & & \\
\hline
\end{tabular}

${ }^{1}$ Difference $(\mathrm{mm})=$ Calc. - Exp., ${ }^{2}$ Difference $(\%)=\frac{\text { Calc. }- \text { Exp. }}{\text { Exp. }}$

graph. It shows excellent agreement until $7 \mathrm{~cm}$ depth in the water phantom with differences of less than $2 \%$, which is totally within the uncertainties in this region. Up to $7.8 \mathrm{~cm}$, i.e. along the very steep distal fall off, the differences are of about $5 \%$, and increase in the tail, but with high uncertainties. In this latter region, the difference between measured and calculated doses is very sensitive to small uncertainties in either the measurement depth or, as far as this model is concerned, the materials of the modulator and their densities, or the angular opening of the modulator steps. Table III presents a quantitative comparison between the calculated and measured range $\left(Z_{d 90}\right)$, modulation $\left(Z_{p 95}-Z_{d 95}\right)$ and penumbra width $\left(Z_{d 80}-Z_{d 20}\right)$. Overall agreement was found for all of these parameters. The maximum difference is $0.4 \%$ and $0.3 \mathrm{~mm}$ for the three studied characteristics. The difference observed between the calculated pristine step 1 (Tab. II) and SOBP $Z_{d 90}$ is due to the different normalisation of both dose distributions, i.e. the pristine step 1 Bragg peak is normalised to its maximum, whereas the SOBP is normalised to the plateau; also, the tail of the pristine step 2 Bragg peak contributes about $5 \%$ to the SOBP at depth $Z_{d 90}$.

\subsubsection{Relative lateral dose profiles}

The calculated and measured lateral profiles in the horizontal $(X)$ and vertical $(Y)$ directions at each of the two depths in water, and the corresponding ratio between calculated and measured distributions, are shown in Figure 11. We should note that the measured profile in the horizontal direction at $7 \mathrm{~cm}$ depth suffers from higher fluctuations due to poorer statistics for this measurement. In the plateau region a good agreement is generally found in the four profiles: the differences are within $3 \%$ except for the profile at $7 \mathrm{~cm}$ depth in the $X$ direction, where they can reach $7 \%$. In the lateral penumbra part, the differences increase in all of the profiles. This 

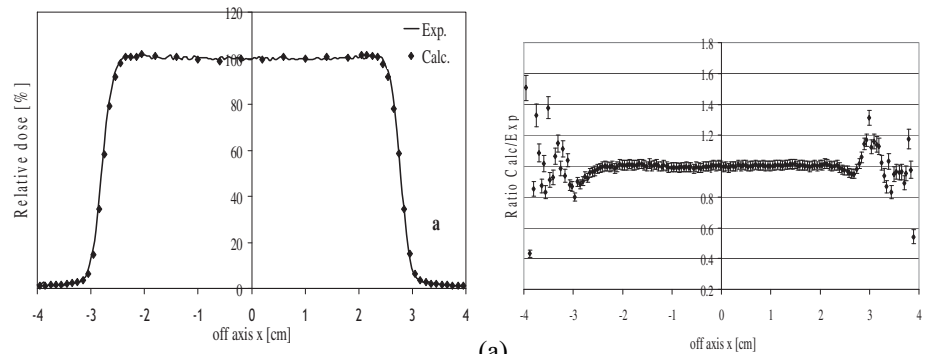

(a)
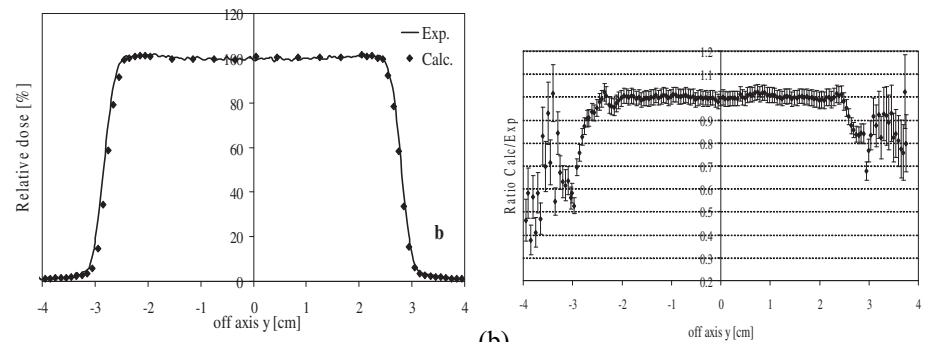

(b)
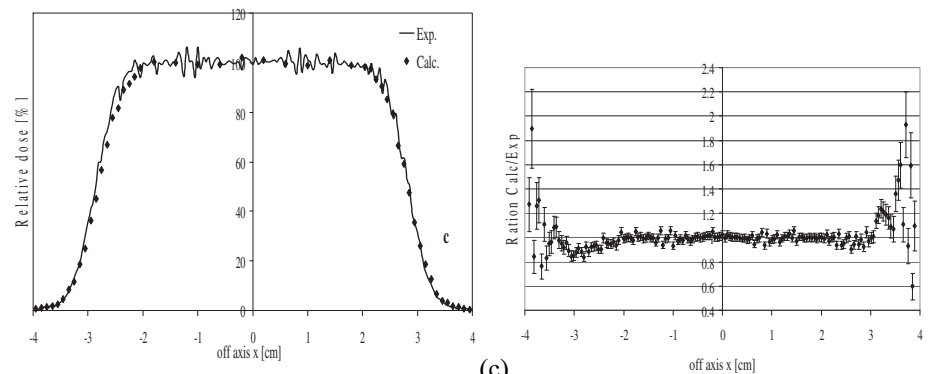

(c)
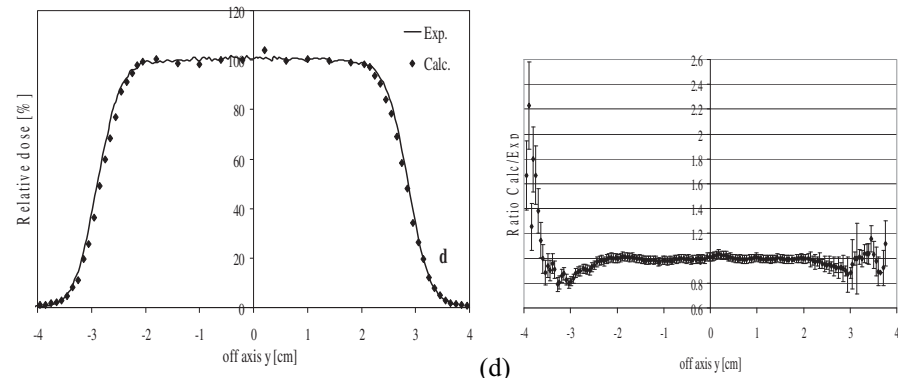

Figure 11 - Relative lateral dose profiles. Left: measured (Exp.) and calculated (Calc.) relative lateral dose profiles. Right: corresponding ratio between calculated and measured lateral distributions. Horizontal (a) and vertical (b) profiles at the depth of $0.5 \mathrm{~cm}$ in water, and horizontal (c) and vertical (d) profiles at the depth of $7 \mathrm{~cm}$ in water are presented. The uncertainties are only shown on the ratio graphs. 
effect was expected because of the strong dose gradients and the higher uncertainties in this region. In the lateral tail region the differences can become higher, reaching a factor of 2 for some profiles but, similarly to arguments developed in paragraph 3.3.2, in this region the difference between measured and calculated doses is very sensitive to any measurement asymmetry or to the lateral $X$ and $Y$ extensions and angular opening of the proton beam at the entrance of the nozzle defined in the model. It is worth mentioning that the correct simultaneous reproduction of the lateral dose profiles at $0.5 \mathrm{~cm}$ and $7 \mathrm{~cm}$ indicate that the multiple Coulomb scattering (MCS) model correctly describes the proton scattering, at least in water. Table IV shows the comparison between calculated and measured field widths $(90 \%-90 \%$ and $50 \%-50 \%)$, and penumbra width $(80 \%-20 \%)$. The differences in field widths are less than $2 \%$, while they can reach $12 \%$ for the penumbra width, but this corresponds to only a $0.6 \mathrm{~mm}$ difference.

Finally, it should be mentioned that the differences found between calculated and measured proton depth dose distributions and lateral profiles are comparable with the results published in similar studies (Paganetti et al., 2004; Hérault et al., 2005; Newhauser et al., 2005; Polf et al., 2007; Koch et al., 2008; Martinetti et al., 2009; Stankovskiy et al., 2009; etc.). Indeed, the MC model presented by Paganetti et al. (2004) accurately reproduced measured dose distributions in range and modulation width, in the case of a spread-out Bragg peak (SOBP), with an agreement of $1 \mathrm{~mm}$. Meanwhile, for Newhauser et al. (2005), the maximum differences between absorbed dose profiles from simulations and measurements were $6 \%$ and $0.6 \mathrm{~mm}$, while typical differences were less than $2 \%$ and $0.2 \mathrm{~mm}$. Finally, for Koch et al. (2008), the Monte Carlo method predicted dose profiles that agreed with measurements to within $3 \%$ of the peak dose or within $0.5 \mathrm{~mm}$ distance-to-agreement. Similar agreements of the order of the millimetre were found by other authors when comparing proton relative depth dose distributions and lateral profiles (considering range, penumbra width, field widths, etc.) calculated using Monte Carlo codes with the corresponding measurement.

\section{Conclusion}

A Monte Carlo model of the IBA gantry at the ICPO was developed using the MCNPX tool. The beamline model is used to treat paediatric patients with intracranial tumours at a specific energy of $178.16 \mathrm{MeV}$. The beamline components and the treatment room were both modelled. The proton beam source was defined on the basis of measurements and IBA data in addition to some calculations where information was not available. The particle transport and interaction physics were carefully defined in MCNPX. The model was validated with a series of comparisons between calculated and measured relative proton dose 
MONTE CARLO SIMULATION OF A PROTON THERAPY BEAMLINE

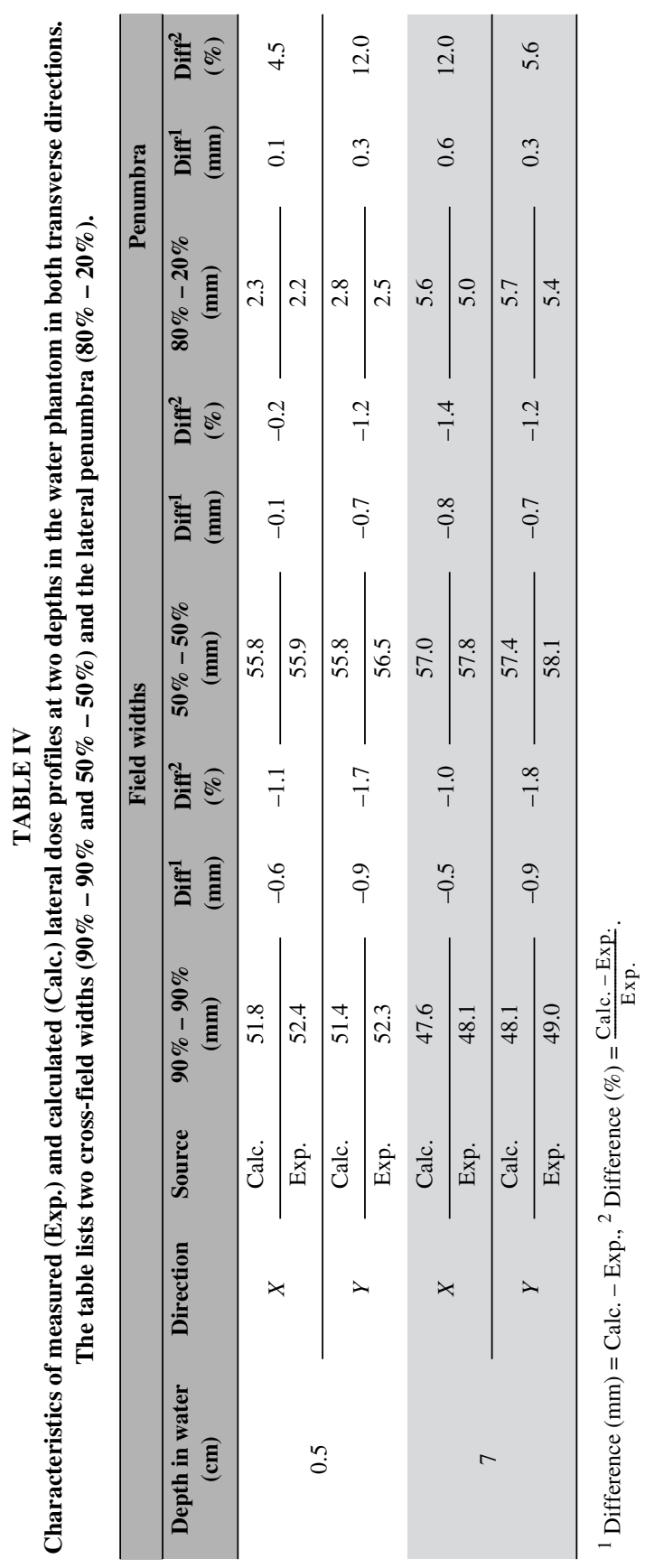


distributions. This included pristine Bragg peaks, the spread-out Bragg Peak and lateral dose profiles in modulated conditions. A comparison between calculated and measured profiles showed an overall good agreement, since a difference of less than $2 \mathrm{~mm}$ was found in all cases. Thus, we can consider that our Monte Carlo model is able to correctly reproduce the proton dose distributions when keeping in mind that the final purpose is to study radiation protection of the patient. In fact, the developed Monte Carlo model is aimed to be used for the evaluation of the secondary neutron doses received by the patient during treatment. Therefore, the model benchmarking against the relative proton dose distributions alone is not sufficient as it does not demonstrate the capability of the model to correctly predict the neutron doses away from the treatment volume. Further experimental comparisons, relating secondary neutron doses and spectra at different locations in the treatment room, and neutron doses inside a physical phantom are necessary. These validation steps, as well as calculations of secondary neutron doses in reference voxel phantoms of different ages, will be presented in a forthcoming paper.

\section{REFERENCES}

Agosteo S., Birattari C., Caravaggio M., Silari M., Tosi G. (1998) Secondary neutron and photon dose in proton therapy, Radiother. Oncol. 48, 293-305.

Bertini H.W. (1968) Intranuclear-cascade calculation of the secondary nucleon spectra for nucleonnucleus interactions, Phys. Rev. 188, 1711-1720.

Bonnet D.E. (1993) Current developments in proton therapy: a review, Phys. Med. Biol. 38, 1393-1401.

Chadwick M.B., Young P.G., MacFarlane R.E., Moller P., Hale G.M., Little R.C., Koning A.J., Chiba S. (1999) LA150 Documentation of Cross Sections, Heating, and Damage: Part A (Incident Neutrons) and Part B (Incident Protons) Los Alamos National Laboratory report LA-UR-991222

Hendricks J.S., McKinney G.W., Durkee J.W., Finch J.P., Fensin M.L., James M.R., Johns R.C., Pelowitz D.B., Waters L.S. (2006) MCNPX, VERSION 26C, LA-UR-06-7991, Los Alamos National Laboratory.

Hérault J., Iborra N., Serrano B., Chauvel P. (2005) Monte Carlo simulation of a protontherapy platform devoted to ocular melanoma, Med. Phys. 32, 910-919.

Hérault J., Iborra N., Serrano B., Chauvel P. (2007) Spread-out Bragg peak and monitor units calculation with the Monte Carlo code MCNPX, Med. Phys. 34, 680-688.

IBA, 2007, Ionization chambers and diode detectors, Detectors for relative and absolute dosimetry, IBA dosimetry.

Jiang H., Wang B., Xu X.G., Suit H.D., Paganetti H. (2005) Simulation of organ-specific patient effective dose due to secondary neutrons in proton radiation treatment, Phys. Med. Biol. 50, 4337-4353.

Martinetti F., Donadille L., Delacroix S., Nauraye C., De Oliveira A., Clairand I., Hérault J. (2009) Monte Carlo Modelling of a protontherapy beamline dedicated to ophthalmologic treatments, Nuclear Technology 168, 721-727. 
Newhauser W.D., Titt U., Dexheimer D., Yan X., Nill S. (2002) Neutron shielding verification measurements and simulations for a $235 \mathrm{MeV}$ proton therapy centre, Nucl. Instrum. Meth. A. 476, 80-84.

Newhauser W.D., Koch N., Hummel S., Ziegler M., Titt U. (2005) Monte Carlo simulations of a nozzle for the treatment of ocular tumours with high-energy proton beams, Phys. Med. Biol. 50, 52295249.

Olsen D.R., Bruland O.S., Frykholm G., Norderhaug I.N. (2007) Proton therapy - A systematic review of clinical effectiveness, Radiother. Oncl. 83, 123-132.

Paganetti H., Jiang H., Lee S.Y., Kooy H.M. (2004). Monte Carlo simulations for nozzle design, commissioning and quality assurance for a proton radiation therapy facility, Med. Phys. 31, 2107-2118.

Paganetti H., Jiang H., Parodi K., Slopsema R., Engelsman M. (2008) Clinical implementation of full Monte Carlo dose calculation in proton beam therapy, Phys. Med. Biol. 53, 4825-4853.

Perez-Andujar A., Newhauser W.D., DeLuca P.M. (2009) Contribution to neutron fluence and neutron absorbed dose from double scattering proton therapy system components, Nucl. Tech. 168, 728735.

Polf J.C., Harvey M.C., Titt U., Newhauser W.D., Smith A.R. (2007) Initial beam size study for passive scatter proton therapy I. Monte Carlo verification, Med. Phys. 34, 4213-4218.

PTCOG (2010) Particule Therapy Co-Ordinated Group, PTCOG Publications Sub-Committee Task Group on Shielding Design and Radiation Safety of Charged Particule Therapy Facilities, PTCOG Report 1.

Rossi B., Greisen K. (1941) Cosmic-ray theory, Rev. Mod. Phys. 13, 240-270.

Sisterson J.M. (1995) Proton radiation therapy: a summary of the world wide experience, Nucl. Instrum. Meth. B 99, 827-829.

Stankovskiy A., Kerhoas-Cavata S., Ferrand R., Nauraye C., Demarzi L. (2009) Monte Carlo modelling of a treatment line of Proton Therapy Center in Orsay, Phys. Med. Biol. 54, 23772394.

Vavilov P.V. (1957) Ionizational losses of high-energy heavy particles, Sov. Phys. JETP 5, 749-751.

White M.C. (2002) Photoatomic Data Library MCPLIB04: A New Photoatomic Library Based on Data from ENDF/B-VI Release 8 Los Alamos National Laboratory internal memorandum X-5: MCW-02-111.

Zacharatou Jarlskog C., Paganetti H. (2008) Risk of developing second cancer from neutron dose in proton therapy as function of field characteristics, organ, and patient age, Int. J. Radiat. Oncol. Bio. Phys. 72, 228-235.

Zheng Y., Fontenot J., Taddei P., Mirkovic D., Newhauser W. (2008) Monte Carlo simulations of neutron spectral fluence, radiation weighting factor and ambient dose equivalent for a passively scattered proton therapy unit, Phys. Med. Biol. 53, 187-201. 